\title{
Medo do parto: Uma revisão das intervenções baseadas na Terapia Cognitivo-Comportamental
}

\section{Lívia R. de C. Nunes', Fernanda C. Coutinho², e Veruska Andréa dos Santos 3}

' Programa de Pós-Graduação em Psicologia Social, Universidade do Estado do Rio de Janeiro (Uerj)

2 Programa de Pós-Graduação Lato Sensu em Terapia Cognitivo-Comportamental, Pontifícia Universidade Católica do Rio de Janeiro (PUC-Rio)

3 Instituto de Psiquiatria (Ipub), Universidade Federal do Rio de Janeiro (UFRJ)

Submissão: 30 set. 2020.

Aceite: 9 ago. 2021.

\section{Notas das autoras}

Lívia R. de C. Nunes (D) https://orcid.org/00oo-0003-1253-159X

Fernanda C. Coutinho (D) https://orcid.org/00oo-0001-6522-2358

Veruska Andréa dos Santos (D) https://orcid.org/0000-0002-8518-5408

Correspondências referentes a este artigo devem ser enviadas para Lívia R. de C. Nunes, Rua São Francisco Xavier, 524,10 $0^{\circ}$ andar, sala 10009, bloco F, Maracanã, Rio de Janeiro, RJ, Brasil. CEP 20550013. E-mail: psilivianunes@gmail.com 


\title{
Resumo
}

O medo do parto vaginal é bastante comum e pode ter impactos relevantes na saúde materno-infantil. Este estudo teve como objetivos investigar de que forma a Terapia Cognitivo-Comportamental (TCC) tem sido aplicada em casos de medo do parto e avaliar a eficácia dessas intervenções. Realizou-se uma revisão sistemática da literatura por meio das bases de dados BVS Brasil, Scopus e PubMed, no período de fevereiro a abril de 2019. Após a análise dos critérios de inclusão e exclusão, um total de seis textos completos constituíram a amostra final. Técnicas terapêuticas como a reestruturação cognitiva, a exposição e a atenção plena demonstraram ser relevantes para a elaboração de uma percepção menos catastrófica do parto e para o desenvolvimento de maior confiança nas próprias habilidades de enfrentamento do trabalho de parto. Conclui-se que as intervenções em TCC apresentam potencial benefício para o tratamento do medo do parto.

Palavras-chave: gravidez, medo, parto, Terapia Cognitivo-Comportamental, tocofobia

\section{FEAR OF CHILDBIRTH: A REVIEW OF INTERVENTIONS BASED ON COGNITIVE BEHAVIORAL THERAPY}

\begin{abstract}
Fear of childbirth is a very common phenomenon among women and may significantly impact maternal-infant health. The goal of this study is to investigate how Cognitive Behavioral Therapy (CBT) has been applied in cases of fear of childbirth and assess the efficacy of such interventions. A systematic review of these practices was made using the BVS Brazil, Scopus, and PubMed databases, from February to April 2019. After analyzing the inclusion and exclusion criteria, a total of six full studies were selected as the final sample for this research. Therapeutic techniques such as cognitive restructuring, exposure, and mindfulness have proven to be important means of developing a less catastrophic perception of childbirth along with greater self-confidence in the abilities to cope with labor. The review led to the conclusion that CBT does have the potential to treat fear of childbirth.
\end{abstract}

Keywords: pregnancy, fear, childbirth, Cognitive Behavioral Therapy, tokophobia

\section{MIEDO AL PARTO: UNA REVISIÓN DE INTERVENCIONES BASADAS EN TERAPIA COGNITIVO-CONDUCTUAL}

\section{Resumen}

El miedo al parto es muy común y puede afectar significativamente la salud materno-infantil. El objetivo de este estudio fue investigar cómo se ha aplicado la Terapia Cognitivo-Conductual (TCC) en casos de miedo al parto y evaluar la eficacia de las intervenciones. Se realizó una revisión sistemática a través de las bases de datos BVS Brasil, Scopus y PubMed, de febrero a abril de 2019. Después de analizar los criterios de inclusión y exclusión, un total de seis textos completos constituyeron la muestra final. Las 
técnicas terapéuticas como la reestructuración cognitiva, la exposición y la atención plena demostraron ser relevantes para la elaboración de una percepción menos catastrófica del parto y para el desarrollo de una mayor confianza en las capacidades propias para enfrentar el parto. La revisión llevó a la conclusión de que la TCC tiene potencial benéfico para tratar el miedo al parto.

Palabras clave: embarazo, miedo, parto, Terapia Cognitivo-Conductual, tocofobia 
Sabe-se que a experiência de parir está acompanhada de um pequeno risco de morbimortalidade materna (World Health Organization [WHO], 2019). No Brasil, em 2017, foram registrados 60 óbitos maternos para cada 100 mil nascidos vivos (WHO, 2019). Dessa forma, algum nível de medo relacionado ao parto é esperado. Contudo, outros fatores como a influência cultural, a desinformação, a mídia, os relatos negativos de outras mulheres e o modelo de assistência obstétrica medicalizante podem exacerbar esse temor (Pereira et al., 2011). Esse modelo de assistência tem como forte característica a relação de poder médico-paciente. Dessa forma, o médico decide quais informações sobre a gestação e o parto serão oferecidas à mulher. Frequentemente, essas informações (ou a ausência delas) facilitam o medo do parto vaginal e reforçam a escolha da cesárea eletiva como uma opção mais prática, indolor e conveniente (Pereira et al., 2011).

O medo do parto pode ser classificado de acordo com a intensidade da emoção. Um nível baixo de medo pode ser compreendido como uma preocupação manejável que ajuda a mulher a se preparar para o momento do parto. Um nível moderado representa uma preocupação difícil de ser tolerada sem suporte, mas que ainda não compromete a saúde mental da mulher. O medo severo pode comprometer a saúde mental e prejudicar a conexão com o bebê. Já o medo fóbico, também conhecido como tocofobia ou partofobia, pode gerar inclusive a evitação da gestação ou do parto, ainda que o bebê seja desejado (Ryding, 2017). Outras formas de evitação incluem esquivar-se de receber informações sobre o trabalho de parto, não participar de grupos de suporte, barrar memórias relacionadas ao medo e optar pela cesariana como via de nascimento (Hofberg \& Brockington, 2000; Rondung et al., 2016).

A palavra tocofobia tem origem no termo grego tocos que significa "parto". Atualmente, os estudos frequentemente se referem à tocofobia como pertencente ao domínio dos transtornos de ansiedade (Hofberg \& Ward, 2003; Rondung et al., 2016). Ela pode ser classificada como tocofobia primária ou secundária. A tocofobia primária costuma ter início na adolescência ou no início da fase adulta e pode ter origem em diferentes fatores, como: a exposição indireta a situações de parto traumáticas ou estressoras; a obtenção de informações negativas sobre o parto; situações de abuso sexual; e a generalização de outros medos - por exemplo fobia de sangue, injeções, hospitais etc. (Hofberg \& Ward, 2003; Rondung et al., 2016).

Já a tocofobia secundária tem como origem principal o condicionamento pelo medo decorrente de um evento obstétrico traumático. Esse evento está mais frequentemente relacionado a um parto traumático, mas também pode ocorrer após um aborto espontâneo, provocado ou em casos de bebê natimorto. Nesses casos, o medo do parto pode ter relação com o transtorno de estresse pós-traumático - TEPT (Hofberg \& Ward, 2003; Rondung et al., 2016). A relação entre partos traumáticos, tocofobia secundária e TEPT é especialmente relevante quando consideramos que $25 \%$ das mulheres relataram ter sofrido algum tipo de vio- 
lência durante o atendimento ao parto no Brasil, segundo os dados de uma pesquisa de opinião pública realizada em 2010 (Venturi et al., 2010).

Há uma estimativa de $14 \%$ de prevalência da tocofobia na população mundial, número que parece ter aumentado do ano 2000 em diante (O'Connell et al., 2017). Contudo, essa estimativa é limitada pela falta de consistência no diagnóstico do transtorno. Em estudo realizado na Finlândia, das 329 grávidas entrevistadas, 78\% apresentaram medo relativo à gestação, ao parto ou a ambos os eventos (Melender, 2002). Outro estudo realizado na Suécia, com 139 grávidas, verificou a presença de medo severo do parto em 9\% das participantes da pesquisa e 17\% apresentaram medo moderado (Areskog et al., 1981). Ainda não há informação sobre a prevalência da tocofobia na população brasileira. Em 2021, Nunes et al. (2021) publicaram o primeiro instrumento de mensuração da fobia do parto validado no país: o Questionário de Avaliação da Partofobia (QAP).

Apesar de ser um tema pouco pesquisado no Brasil, o medo da dor do parto é apontado como uma das principais motivações para a escolha de uma cesárea eletiva (Pereira et al., 2011). Um dos motivos é a ilusão de controle que a cirurgia oferece à mulher (Bewley \& Cockburn, 2002). O grande número de cesarianas realizadas no país recebeu grande atenção com os resultados da pesquisa Nascer no Brasil (Leal et al., 2012). Das 23.894 mulheres entrevistadas em território nacional, $52 \%$ tiveram seus filhos por meio de partos cesáreos. Quando se analisou apenas o setor privado, constatou-se que $88 \%$ dos nascimentos foram por cesariana, número ainda mais preocupante, visto que cirurgias desse tipo envolvem riscos imediatos e de longo prazo (Organização Mundial de Saúde, 2015). Em contrapartida, aproximadamente $70 \%$ das mulheres brasileiras desejavam um parto vaginal no início da gravidez. No entanto, poucas foram apoiadas em suas escolhas iniciais.

A diferença entre a quantidade de mulheres que almejam o parto vaginal e a quantidade de mulheres que de fato o realizam pode estar relacionada à falsa ideia de que a cesariana seria mais segura para a mulher (mesmo sem uma indicação médica real) e à falta de informação oferecida à gestante durante o pré-natal. Como consequência, o poder de autonomia das muIheres é prejudicado pela impossibilidade de tomar uma decisão informada (Pereira et al., 2011).

As mulheres submetidas à cirurgia cesariana sem indicação obstétrica real (Souza et al., 2010) são expostas, com seus filhos, a riscos de morbidade e mortalidade desnecessários (Leal et al., 2012). Nesse sentido, existe um conflito ético em realizar a cesárea eletiva em mulheres que a solicitam por medo do parto sem antes disponibilizar atenção e suporte psicológico. Se, ainda assim, mesmo após receber o apoio psicológico, a mulher optar pela cirurgia cesariana por medo severo do parto, negar a cirurgia eletiva seria cruel (Bewley \& Cockburn, 2002). Em todo caso, oferecidos os recursos necessários para uma escolha consciente, é importante que seja respeitado o desejo da mulher, em conformidade com seus direitos reprodutivos e sexuais (Zorzam \& Cavalcanti, 2017). Em estudo realizado por Hofberg e Brockington 
(2000), a negação da via de parto escolhida pela mulher esteve associada ao desenvolvimento do TEPT após o parto.

O suporte e a atenção psicológica oferecidos às mulheres com medo severo do parto se mostram ainda mais fundamentais quando consideramos o ciclo vicioso do medo do parto. Mulheres com altos níveis pré-natais de medo do parto estão suscetíveis a um risco aumentado de sentir medo durante o trabalho de parto e perceber a situação como ameaçadora. Essas circunstâncias podem aumentar as chances de uma experiência de nascimento negativa, que pode ter como consequência o medo relacionado a um parto futuro (Rondung et al., 2016). Contudo, intervenções terapêuticas focadas no medo do parto divergem quanto aos métodos de avaliação, conceitualização e aplicação das intervenções (Striebich et al., 2018). Dentre essas, a Terapia Cognitivo-Comportamental (TCC) tem sido investigada no tratamento do medo de parir (Striebich et al., 2018).

A TCC é uma abordagem de psicoterapia composta por sessões estruturadas e com duração limitada. A abordagem se baseia no modelo cognitivo, segundo o qual cognições distorcidas ou disfuncionais (que afetam o humor e o comportamento do paciente) são comuns a todos os transtornos psicológicos. Nesse sentido, a avaliação realista e as mudanças no pensamento produzem uma melhora no humor e no comportamento (Beck, 2007). Além da identificação e reestruturação de pensamentos disfuncionais, a abordagem também engloba técnicas que auxiliam a regulação emocional e a mudança comportamental. Entre as técnicas utilizadas, podemos citar a psicoeducação, a confrontação empática, a exposição e o relaxamento (Beck, 2007).

Ghazaie et al. (2016) realizaram um estudo clínico randomizado com 25 mulheres e concluíram que a TCC é capaz de diminuir cesáreas desnecessárias por meio da redução do medo do parto, da redução do medo da dor e do aumento da percepção de autoeficácia. Após a revisão sistemática realizada por Striebich et al. (2018) sobre a utilização de diversos tipos de terapia no manejo do medo do parto, esta revisão pode contribuir dando foco à utilização da TCC. Dessa forma, o objetivo desta revisão é investigar de que forma a TCC tem sido aplicada em casos de medo do parto e quais pesquisas têm avaliado a sua eficácia. Espera-se que o estudo contribua para melhor compreensão dos processos psicológicos em mulheres com medo do parto e ajude a identificar as melhores formas de tratamento e prevenção baseadas na TCC.

\section{Método}

Como apontado por Sampaio e Mancini (2007), a revisão sistemática utiliza como fonte de dados a literatura existente sobre determinado tema e tem o intuito de resumir e apresentar as evidências relacionadas a uma intervenção específica. Esse processo se dá por meio da aplicação de métodos explícitos e sistematizados de busca, avaliação crítica e sintetização das informações selecionadas (Sampaio \& Mancini, 2007). 


\section{Critérios de inclusão do material}

Os critérios de inclusão da busca foram: texto completo em inglês ou português; estudos que incluem mulheres (grávidas ou não) com medo do parto; estudos que tiveram como intervenção a TCC ou práticas de mindfulness. Optou-se por incluir os estudos cujas intervenções foram baseadas em mindfulness, devido à ampla utilização das práticas de atenção plena em diferentes terapias comportamentais e cognitivas contemporâneas (Vandenberghe \& Sousa, 2006). Já os critérios de exclusão foram: revisões de literatura ou comentários; estudos não focados no medo do parto; estudos que não tiveram como intervenção a TCC ou práticas de mindfulness. Não houve restrição quanto ao período de publicação dos estudos.

\section{Procedimentos de coleta de dados documentais}

As questões de pesquisa que nortearam este estudo foram: Como a TCC tem sido aplicada em casos de medo do parto? Essas intervenções têm se mostrado eficazes? A pesquisa foi realizada por meio das bases de dados BVS Brasil, Scopus, e PubMed, no período de fevereiro a abril de 2019. Os seguintes descritores e operadores booleanos foram utilizados: "Cognitive Therapy" OR "Cognitive behavioral therapy" OR "CBT" AND "fear of birth" OR "fear of childbirth" OR "tokophobia". Essa combinação foi escolhida por meio de uma articulação entre o controle de vocabulário do Medical Subject Headings (MeSH), os Descritores em Ciências da Saúde (DeCS) e os descritores utilizados por Striebich et al. (2018) em sua revisão sistemática sobre intervenções para o medo do parto. Assim sendo, a combinação dos termos apresentados teve como intenção focar ao máximo o tema de interesse deste trabalho.

\section{Procedimentos de análise}

Para a análise qualitativa dos resultados, os estudos foram categorizados a partir de título, autoria, ano de publicação, país de origem, objetivo do estudo, desenho do estudo, público-alvo, instrumento utilizado na avaliação do medo do parto, técnica de intervenção e eficácia do tratamento.

\section{Resultados}

A princípio foram identificados 30 artigos. Após a remoção dos duplicados, obteve-se uma seleção de 16 artigos, dos quais oito foram excluídos em conformidade com os critérios de inclusão e exclusão desta revisão. Dessa forma, um total de oito textos completos foram obtidos, dos quais dois foram removidos por representarem protocolos de estudos. Finalmente, incluíram-se seis artigos na síntese qualitativa. O número total de estudos selecionados e eliminados em cada uma das etapas do processo de avaliação sistemática pode ser observado no fluxograma do processo de avaliação (Figura 1), conforme as recomendações do Preferred Reporting Items for Systematic Reviews and Meta-Analyses - PRISMA (Moher et al., 2009). 
Figura 1

Diagrama das estratégias metodológicas utilizadas para seleção dos artigos

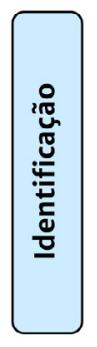

Relatos identificados nas bases de dados de pesquisa $(n=30)$

Scopus $(n=14)$

PubMed $(n=9)$

$\operatorname{BVS}(n=7)$

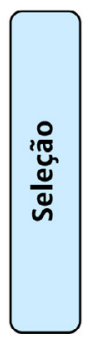

Relatos após eliminação dos duplicados $(n=16)$
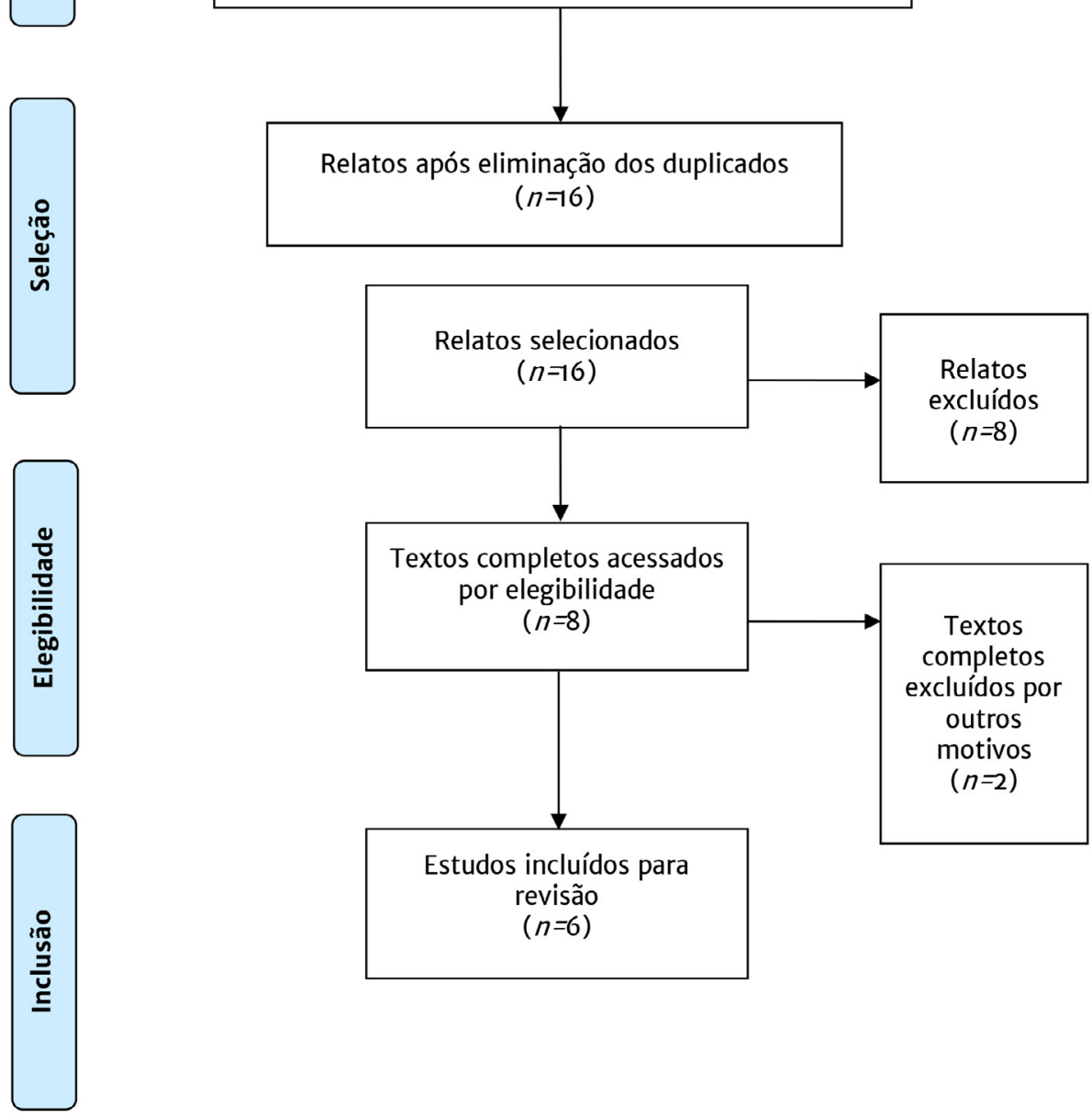

Estudos incluídos para revisão $(n=6)$

Nota. Fonte: Moher et al. (2009). 
Como é possível observar na Tabela 1, dos seis artigos selecionados, quatro são suecos, um é australiano e o outro é turco. Todos os artigos foram publicados em inglês, no período de 2014 a 2018.

Tabela 1

Caracterização dos estudos

\begin{tabular}{|c|c|c|c|c|c|c|}
\hline $\begin{array}{l}\text { Autor } \\
\text { (ano) }\end{array}$ & País & $\begin{array}{c}\text { Desenho } \\
\text { do estudo }\end{array}$ & $\begin{array}{c}\text { População- } \\
\text {-alvo }\end{array}$ & Instrumento & Intervenção & Formato \\
\hline $\begin{array}{l}\text { Byrne } \\
\text { et al. (2014) }\end{array}$ & Austrália & $\begin{array}{l}\text { Estudo } \\
\text { pré-experi- } \\
\text { mental de pré } \\
\text { e pós-teste } \\
\text { com um grupo }\end{array}$ & $\begin{array}{l}\text { Mulheres } \\
\text { nulíparas }^{\mathrm{b}}\end{array}$ & $\begin{array}{l}\text { W-DEQ- } \\
-A \text { e W-DEQ-B }\end{array}$ & MBCE & Grupo \\
\hline $\begin{array}{l}\text { Nieminen } \\
\text { et al. (2015) }\end{array}$ & Suécia & $\begin{array}{l}\text { Estudo } \\
\text { qualitativo }\end{array}$ & $\begin{array}{l}\text { Mulheres } \\
\text { nulíparas com } \\
\text { medo do parto } \\
(\text { W-DEQ } \geq 85)\end{array}$ & W-DEQ-A & iTCC & Individual \\
\hline $\begin{array}{l}\text { Nieminen } \\
\text { et al. (2016) }\end{array}$ & Suécia & $\begin{array}{l}\text { Estudo } \\
\text { pré-experi- } \\
\text { mental de pré } \\
\text { e pós-teste } \\
\text { com um grupoa }\end{array}$ & $\begin{array}{l}\text { Mulheres } \\
\text { nulíparas com } \\
\text { medo do parto } \\
(\text { W-DEQ } \geq 85 \text { ) }\end{array}$ & $\begin{array}{l}\text { W-DEQ- } \\
-A \text { e W-DEQ-B }\end{array}$ & iTCC & Individual \\
\hline $\begin{array}{l}\text { Larsson } \\
\text { et al. (2017) }\end{array}$ & Suécia & $\begin{array}{l}\text { Estudo } \\
\text { experimental } \\
\text { verdadeiro }\end{array}$ & $\begin{array}{l}\text { Mulheres } \\
\text { grávidas com } \\
\text { medo do parto } \\
(\text { FOBS } \geq 60 \text { ) }\end{array}$ & FOBS & iTCC & Individual \\
\hline $\begin{array}{l}\text { Rondung } \\
\text { et al. (2018) }\end{array}$ & Suécia & $\begin{array}{l}\text { Estudo } \\
\text { experimental } \\
\text { verdadeiro }\end{array}$ & $\begin{array}{l}\text { Mulheres } \\
\text { grávidas com } \\
\text { medo do parto } \\
\text { (FOBS } \geq 60 \text { ) }\end{array}$ & FOBS & iTCC & Individual \\
\hline $\begin{array}{l}\text { Uçar e } \\
\text { Golbasi } \\
\text { (2018) }\end{array}$ & Turquia & $\begin{array}{l}\text { Estudo quase } \\
\text { experimental }\end{array}$ & $\begin{array}{l}\text { Mulheres } \\
\text { primigestas }\end{array}$ & W-DEQ-A & $\begin{array}{l}\text { Programa } \\
\text { educacional } \\
\text { baseado na TCC }\end{array}$ & Grupo \\
\hline
\end{tabular}

Nota. ${ }^{\text {a }}$ Os autores classificaram como estudo de coorte prospectivo e longitudinal. ${ }^{\mathrm{b}}$ Os acompanhantes das mulheres também receberam a intervenção.

\section{Instrumento utilizado na avaliação do medo do parto}

$O$ instrumento utilizado com mais frequência foi o Wijma Delivery Expectancy-Experience Questionnaire - W-DEQ (Wijma et al., 1998). Desenvolvido por um grupo de suecos, o instrumento é composto de 33 itens avaliados por uma escala Likert de seis pontos. O questionário foi elaborado com a intenção de medir o medo do parto por meio das avaliações cognitivas da mulher a respeito do parto (Wijma et al., 1998). O instrumento mede tanto o medo relacionado ao parto durante a gestação quanto o medo relacionado ao parto durante o puerpério, por meio de perguntas a respeito das expectativas quanto ao parto (versão A) e 
experiências após o parto (versão B) (Wijma et al., 1998). O ponto de corte $\geq 85$ é considerado adequado para a identificação do medo severo do parto (Nieminen et al., 2016)

Outro instrumento utilizado foi a Fear of Birth Scale - FOBS (Ternström, 2018), uma escala visual analógica que aborda dois constructos: medo e preocupação. Os respondentes são convocados a avaliar seus sentimentos a respeito do parto que está por vir respondendo à pergunta "How do you feel right now about the approaching birth?", que poderia ser traduzida por "Como você se sente neste exato momento a respeito do parto que está por vir?". A resposta é dada por meio de uma escala visual analógica ancorada pelas palavras "calm/worried", que podem ser traduzidas por "calma/preocupada", e "no fear/strong fear", que podem ser traduzidas por "sem medo/muito medo". A pontuação pode variar de o a 100, e quanto maior a pontuação, maior o medo do parto. A pontuação utilizada como pontuação de corte para identificar o medo do parto é 60 (Ternström, 2018). Ao contrário dos demais estudos, o estudo de Byrne et al. (2014) e de Uçar e Golbasi (2018) não tinham como critério de inclusão que as mulheres fossem identificadas com medo do parto/fear of birth (FOB). Dessa forma, o instrumento de avaliação do medo do parto foi utilizado no início do estudo, mas não houve nenhum ponto de corte estabelecido referente à pontuação no instrumento.

\section{Técnicas de intervenção}

Quanto às técnicas de intervenção, a mais utilizada foi a Terapia Cognitivo-Comportamental pela Internet (iTCC). A iTCC costuma ser aplicada por meio de uma plataforma on- line que disponibiliza módulos autoguiados com materiais psicoeducacionais e exercícios da TCC (Berger, 2016). As plataformas também permitem que o terapeuta se comunique com o paciente de forma privada e confidencial, e acompanhe seu desenvolvimento durante o tratamento (Berger, 2016). Além da versão on-line individual, a TCC também foi aplicada de forma presencial por Uçar e Golbasi (2018) em encontros grupais com um número de cinco a nove pessoas.

Outra intervenção utilizada pelo estudo de Byrne et al. (2014) foi a Mindfulness-Based Childbirth Education (MBCE), que pode ser traduzida por "Educação pré-natal baseada em atenção plena". A MBCE foi desenvolvida especialmente para o estudo e aplicada em grupos de mulheres que poderiam estar acompanhadas por uma figura de suporte (companheiro, companheira, mãe, amiga etc.) que também recebeu a intervenção. A Tabela 2 especifica as técnicas utilizadas em cada intervenção. Algumas técnicas como "técnicas de respiração" e "técnicas de relaxamento" costumam estar presentes em exercícios de atenção plena, mas só foram consideradas quando os autores fizeram menção direta a elas. 
Tabela 2

Técnicas utilizadas em cada um dos protocolos de intervenção dos seis artigos analisados

\begin{tabular}{|c|c|c|c|c|c|c|}
\hline \multirow[t]{2}{*}{ Técnica } & \multicolumn{6}{|c|}{ Autor (ano) } \\
\hline & $\begin{array}{l}\text { Byrne } \\
\text { et al. } \\
(2014)\end{array}$ & $\begin{array}{c}\text { Nieminen } \\
\text { et al. } \\
(2015)\end{array}$ & $\begin{array}{c}\text { Nieminen } \\
\text { et al. } \\
(2016)\end{array}$ & $\begin{array}{c}\text { Larsson } \\
\text { et al. } \\
(2017)\end{array}$ & $\begin{array}{c}\text { Rondung } \\
\text { et al. } \\
(2018)\end{array}$ & $\begin{array}{c}\text { Uçar } \\
\text { e Golbasi } \\
(2018)\end{array}$ \\
\hline Psicoeducação & & $x$ & $x$ & $x$ & $x$ & $x$ \\
\hline $\begin{array}{l}\text { Reestruturação } \\
\text { cognitiva }\end{array}$ & & $\mathrm{x}$ & $\mathrm{x}$ & $x$ & $x$ & \\
\hline $\begin{array}{l}\text { Treinamento para } \\
\text { tomada de decisão }\end{array}$ & $\mathrm{x}$ & & & & & \\
\hline Exposição & & $x$ & $x$ & $x$ & $x$ & \\
\hline $\begin{array}{l}\text { Técnicas de } \\
\text { respiração }\end{array}$ & & $\mathrm{x}$ & $\mathrm{x}$ & & & $\mathrm{x}$ \\
\hline $\begin{array}{l}\text { Técnicas de } \\
\text { relaxamento }\end{array}$ & & & & & & $x$ \\
\hline $\begin{array}{c}\text { Atenção } \\
\text { plena/meditação }\end{array}$ & $x$ & $x$ & $x$ & $x$ & $x$ & \\
\hline Educação perinatal & $\mathrm{x}$ & $\mathrm{x}$ & $\mathrm{x}$ & & & $\mathrm{x}$ \\
\hline
\end{tabular}

\section{Eficácia do tratamento}

As publicações de Larsson et al. (2017) e Rondung et al. (2018) fazem parte de um mesmo projeto de pesquisa. Cada publicação avalia a mesma intervenção relacionada a desfechos diferentes. O estudo de Larsson et al. (2017) teve como objetivo investigar as preferências pela via de nascimento durante a gravidez e após o parto em mulheres randomizadas para tratamento com iTCC ou aconselhamento para o medo do parto (tratamento padrão oferecido para a população estudada). Houve uma queda na preferência pela cesariana em ambos os grupos desde o início da investigação até a $36^{\mathrm{a}}$ semana de gravidez. No entanto, duas semanas após o parto, a preferência pela cesárea cresceu novamente nos dois grupos, não havendo mudança estatisticamente significativa ao decorrer do tempo no que se refere à preferência pela forma de nascimento. Além disso, as mulheres que receberam aconselhamento padrão se mostraram mais satisfeitas com o tratamento do que aquelas que receberam iTCC.

Algumas informações, como o protocolo de tratamento utilizado na intervenção do tipo iTCC e a quantidade de participantes do grupo de intervenção que concluíram o tratamento, só puderam ser acessadas por meio da publicação de Rondung et al. (2018). Nessa publicação, os autores realizaram uma comparação entre a eficácia do tratamento do tipo iTCC e a eficácia do tratamento do tipo aconselhamento padrão sobre os níveis de medo do parto. O medo do parto não diferiu entre os dois grupos de tratamento após a intervenção. Um ano 
após o parto, as participantes que receberam TCC on-line exibiram níveis de medo do parto significativamente menores.

O protocolo de tratamento por iTCC utilizado em ambos os estudos, como observado na publicação de Rondung et al. (2018), era composto por nove módulos dos quais um era aplicado no follow-up pós-parto. Das 127 mulheres alocadas no grupo que recebeu a intervenção, apenas 13 chegaram a concluir os cinco primeiros módulos. Mais da metade das participantes (53\%) concluiu apenas o primeiro módulo. Assim sendo, a pouca aderência ao tratamento pode ter afetado consideravelmente os resultados encontrados, já que o cálculo do tamanho da amostra sugeria um total de 200 participantes (100 por grupo) para detectar uma redução de $20 \%$ do medo do parto, com um poder de 0,80 e nível de significância de $5 \%$. Dessa forma, o resultado apresentado por Rondung et al. (2018) é difícil de ser interpretado em razão da pouca aderência ao tratamento e da grande variedade de potenciais variáveis mediadoras, moderadoras e confundidoras durante o parto e no período pós-parto.

Larsson et al. (2017) sugerem que as transformações emocionais e cognitivas da gravidez possam não compor um período propício para o aprendizado de novas estratégias de enfrentamento. Entretanto, é possível que a baixa aderência ao tratamento esteja relacionada à baixa motivação das participantes em manejar o medo do parto. Nos estudos de Byrne et al. (2014), Nieminen et al. (2015) e Nieminen et al. (2016), as pesquisas foram divulgadas, e as participantes se inscreveram de forma espontânea nos projetos. Já nos estudos de Larsson et al. (2017) e Rondung et al. (2018), as participantes foram diretamente solicitadas a fazer parte da pesquisa.

O objetivo do estudo de Byrne et al. (2014) foi testar a viabilidade e a eficácia da MBCE na promoção de saúde mental em mulheres grávidas. Foram recrutadas 18 mulheres, e todas concluíram o programa. Contudo, os dados estavam disponíveis de forma completa para apenas 12 dessas, cujas informações foram analisadas. As variáveis "autoeficácia relacionada ao parto" e "medo do parto" foram adequadamente ajustadas para o poder esperado do estudo, já que uma amostra mínima de quatro participantes era necessária. Observou-se uma redução do medo do parto significativa após o tratamento, com um tamanho de efeito grande ( $d$ de Cohen $=1,71$ ), assim como aumento significativo da autoeficácia relacionada ao parto, com tamanho do efeito também grande ( $d$ de Cohen $=-1,91$ ). A ausência de um grupo controle $e$ a pequena amostra utilizada são alguns dos fatores que impossibilitam atribuir os resultados observados à intervenção do tipo MBCE. No entanto, os tamanhos de efeito muito grandes associados às melhorias na autoeficácia e no medo do parto sugerem que esses efeitos possam se relacionar de alguma forma à intervenção e não a efeitos casuais ou processos naturais, como a regressão à média (Byrne et al., 2014).

O estudo de Uçar e Golbasi (2018) teve como objetivo determinar o efeito de uma intervenção educacional baseada na TCC sobre o medo do parto. Foram selecionadas 111 
participantes das quais 52 fizeram parte do grupo de intervenção e 59 do grupo controle que não recebeu nenhum tipo de tratamento. Como apontado pelos autores, na parte final do estudo, o parto de todas as mulheres foi monitorado. A primeira autora não só observou todos os partos, como também participou ativamente no parto das mulheres que eram parte do grupo de intervenção, lembrando-as das informações recebidas durante o programa e oferecendo-Ihes suporte para aplicar os métodos de enfrentamento da dor aprendidos (Uçar \& Golbasi, 2018).

Segundo as autoras, a dor do parto foi menor, o segundo estágio do parto foi mais curto, e o nascimento foi mais satisfatório para o grupo que recebeu a intervenção do que para o grupo controle $(p<0,05)$. Não se informou o tamanho do efeito, mas foi calculado a partir dos dados fornecidos pelas autoras: média, desvio padrão e número de mulheres em cada grupo. A magnitude da diferença entre os grupos após a intervenção foi alta para a dor do parto ( $d$ de Cohen $=1,19)$, média para a duração do segundo estágio do trabalho de parto ( $d$ de Cohen $=0,48$ ) e média para a satisfação relacionada ao nascimento ( $d$ de Cohen $=0,63$ ).

A intervenção da primeira autora, durante o trabalho de parto, torna difícil a atribuição desses resultados ao programa educacional baseado na TCC. A intervenção terapêutica durante o trabalho de parto não faz parte das sessões de TCC, o que aumenta consideravelmente as variáveis de confusão. No fim, as autoras constataram que o medo do parto diminuiu no grupo de intervenção e aumentou no grupo controle $(p<0,001)$. O tamanho do efeito também não foi informado, mas quando calculado mostrou-se grande ( $d$ de Cohen $=1,03$ ).

As pesquisas de Nieminen et al. (2015) e Nieminen et al. (2016) também fazem parte de um mesmo projeto de pesquisa. O estudo teve como objetivo avaliar a viabilidade da iTCC para o tratamento de mulheres com medo severo do parto ( $W-D E Q \geq 85$ ). As mulheres foram recrutadas por uma página na internet que explicava o projeto e por meio da qual elas puderam se cadastrar para participação. Das 42 mulheres que se cadastraram no site, 30 finalizaram o formulário de inscrição e cumpriam os critérios de inclusão da pesquisa. Dessas, 15 completaram todos os oito módulos de tratamento estabelecidos pelo projeto. Na pesquisa de 2015, foram analisadas as narrativas das mulheres a respeito do parto iminente em dois momentos (antes do tratamento e oito semanas após o tratamento). Para coletar as narrativas, as mulheres foram convocadas a realizar um exercício de visualização no qual elas deveriam imaginar e descrever situações específicas relacionadas ao parto. Os dados coletados antes e depois do tratamento foram analisados separadamente por meio de análise temática.

Antes do tratamento, as mulheres relataram principalmente sentimentos de ansiedade, incerteza, solidão, desesperança, falta de autoconfiança e utilização de estratégias de evitação como forma de enfrentamento. O parceiro foi associado a uma ideia de instabilidade, ao passo que os profissionais eram percebidos como pouco atenciosos ou prestativos. Após o tratamento, as mulheres relataram um misto de dúvida e esperança, e uma visão de si mesmas 
como um sujeito ativo e preparado. O parceiro também passou a ser visto como uma pessoa ativa e uma figura de suporte, assim como os profissionais passaram a ser vistos como presentes, prestativos e disponíveis.

Como descrito por Nieminen et al. (2015), o material coletado antes e depois da intervenção foi analisado separadamente, e, dessa forma, não houve comparação entre o antes e o depois de cada indivíduo. Em vez disso, realizou-se uma avaliação dos principais domínios temáticos apresentados. Para evitar um viés de desejo social, as participantes tiveram contato com o terapeuta apenas pela internet. Além disso, o exercício imaginativo realizado após o tratamento ocorreu em um momento em que as participantes já não entravam mais em contato virtual com o terapeuta. O estudo foi relatado como uma pesquisa descritiva e exploratória, e, por isso, não é possível afirmar causalidade entre a terapia e os resultados encontrados. Ainda assim, mostra-se relevante para o aprofundamento de novas investigações.

Já a pesquisa de 2016 contém uma análise quantitativa na qual o medo do parto foi avaliado antes do tratamento, durante o tratamento e após o nascimento do bebê. O medo do parto avaliado antes do tratamento e na última semana de tratamento antes do parto diminuiu significativamente $(p<0,0005)$, e o tamanho do efeito encontrado foi grande ( $d$ de Cohen $=0,95)$. Segundo os autores, a correção post hoc de Bonferroni revelou que a diminuição começou após o módulo três do tratamento, o que corresponde à introdução das tarefas de exposição (Nieminen et al., 2016).

Das 24 mulheres que puderam ser contatadas para o acompanhamento pós-parto, 22 se mostraram satisfeitas com o programa de intervenção e com sua capacidade de auxiliá-las durante a gravidez e o parto. Assim sendo, concluiu-se que o nível de medo do parto mensurado com o $\mathrm{W}$-DEQ é reduzido quando um grupo de mulheres nulíparas motivadas são tratadas com iTCC. No entanto, trata-se de um estudo pré-experimental, e os resultados precisam ser confirmados por estudos clínicos randomizados.

\section{Discussão}

A partir da análise das pesquisas apresentadas, não foi possível chegar a uma conclusão a respeito da eficácia da TCC na redução do medo do parto. Todavia, por se mostrar a TCC tão eficaz no tratamento dos transtornos de ansiedade (Olatunji et al., 2010), novos estudos merecem ser realizados. O estabelecimento de um tratamento eficaz na redução do medo do parto poderia beneficiar o atendimento oferecido às gestantes brasileiras. A ausência de diretrizes específicas para o manejo do medo do parto é grave se consideramos a associação entre medo do parto e cirurgia cesariana (Leal et al., 2012; Pereira et al., 2011). Dessa forma, oferecer um suporte consistente e baseado em evidências científicas para o tratamento do medo do parto, ainda que o medo não seja percebido como fóbico, parece ser uma questão importante de saúde pública por endereçar as grandes taxas de cesáreas eletivas. 
No Brasil, a assistência padrão para o atendimento pré-natal de baixo risco tem como recomendação que no terceiro trimestre a mulher seja informada sobre as diferentes etapas da evolução da gestação e do parto: contrações, dilatação, perda do tampão mucoso, rompimento da bolsa e pós-parto (Brasil, 2012). Além disso, recomenda-se que o profissional de atenção primária à saúde prepare a gestante para o parto vaginal e ajude a diminuir sua ansiedade, insegurança, o medo do parto, da dor, de que o bebê nasça com problemas, entre outras questões emocionais (Brasil, 2012). Apesar das recomendações de uma escuta atenta e um acolhimento por parte do profissional de saúde, não existe uma recomendação objetiva de como essa ajuda deve ser implementada.

Devido à relevância do tema, os pesquisadores suecos têm demonstrado grande interesse em definir, mensurar e tratar o medo do parto. A intervenção do tipo iTCC utilizada por Larsson et al. (2017) e Rondung et al. (2018) foi inspirada pelo protocolo unificado para tratamento transdiagnóstico de transtornos emocionais, pelos manuais anteriores de iTCC para problemas de ansiedade e pela terceira onda de tratamentos com TCC. A utilização do protocolo transdiagnóstico é bastante coerente, visto que o medo do parto está em processo de definição e parece estar associado tanto com a ansiedade quanto com a depressão (Rondung et al., 2016).

Os protocolos utilizados nos seis estudos apresentados abordam diferentes técnicas cognitivas e comportamentais. A educação perinatal é uma delas e parece ser de grande importância para que a mulher tenha conhecimento da fisiologia da gestação e do parto, conheça a capacidade do próprio corpo em lidar com o trabalho de parto e confronte mitos por meio de uma aquisição de conhecimento baseado em evidências. Dos estudos apresentados, os artigos de Larsson et al. (2017) e Rondung et al. (2018) são os únicos cujo protocolo não faz menção direta à educação perinatal. A educação perinatal tem o potencial de auxiliar o manejo de crenças disfuncionais a respeito do parto por fornecer material útil para a apresentação de evidências contra e a favor, facilitando a reestruturação cognitiva.

Outra técnica bastante utilizada foi a exposição, como observado nos estudos de Nieminen et al. (2015), Nieminen et al. (2016), Larsson et al. (2017) e Rondung et al. (2018), e aparentara ser de especial importância no estudo de Nieminen et al. (2016). Essa relevância é coerente com os estudos relacionados ao tratamento de fobias específicas, cabendo ressaltar que as pesquisas de Nieminen et al. (2015) e Nieminen et al. (2016) foram as únicas a utilizar como critério de inclusão a identificação de medo severo do parto nas participantes, o que pode estar mais próximo da intensidade de medo relacionada a uma fobia específica.

Segundo a American Psychological Association - APA (2016), as fobias específicas são mantidas devido à evitação do estímulo fóbico que faz com que o indivíduo não tenha a oportunidade de desenvolver a tolerância ao próprio medo. Ao se expor, o indivíduo tem a oportunidade de observar que suas sensações fisiológicas diminuem com o tempo e que os resultados 
temidos não se concretizam ou não são tão ruins quanto se imagina (APA, 2016). A evitação pode ocorrer pela não inserção em uma situação ou pela inserção incompleta, utilizando recursos como distração (APA, 2016). Dessa forma, as terapias de exposição são elaboradas para encorajar o indivíduo a se engajar nas situações temidas (in vivo ou por meio de exercícios de imaginação) e tentar permanecer nessas situações (APA, 2016). No caso do medo relacionado ao parto, as técnicas de exposição podem incluir ouvir relatos de parto de outras mulheres, assistir a filmes de partos ou observar imagens relacionadas ao parto (Nieminen et al., 2016; Rondung et al., 2018).

Os treinos de atenção plena ou de meditação estiveram presentes em cinco dos seis estudos apresentados. A atenção plena já foi apontada como uma opção para o tratamento da dor crônica (Kabat-Zinn et al., 1986), e seus benefícios têm potencial para serem expandidos também para o parto. A sensação de ser tomada pela dor, o medo de não conseguir enfrentar a dor e o medo de perder o controle e de não ter um bom desempenho são frequentemente reportados em relação ao trabalho de parto (Hofberg \& Ward, 2003; Hughes et al., 2009; Rondung et al., 2016; Pereira et al., 2011). Hughes et al. (2009) sugerem que as práticas meditativas possam ajudar as mulheres na conexão com o momento presente e na consciência das sensações corporais. Dessa forma, espera-se que tais práticas auxiliem no enfrentamento das intensas sensações do trabalho de parto sem julgamento ou autodepreciação caso a muIher opte pela analgesia (Hughes et al., 2009).

Os estudos de Larsson et al. (2017) e Rondung et al. (2018) estabeleceram também, de forma direta, a reestruturação de cognições catastróficas como estratégia de tratamento. Segundo Rondung et al. (2016), os conceitos cognitivos de autoeficácia, intolerância à incerteza e catastrofização da dor parecem ser relevantes para a compreensão do medo do parto. Gatchel et al. (2007) definem a catastrofização da dor como uma orientação negativa e exagerada para a experiência de dor atual ou esperada. Segundo os autores, estudos transversais têm demonstrado que a catastrofização está associada a um aumento da dor e a disfunções psicológicas e físicas em populações clínicas e não clínicas. Os modelos de evitação do medo são multifacetados e possuem componentes afetivos (medo) e comportamentais (evitação), contudo as cognições são identificadas como o cerne da inserção em um ciclo negativo de dor (Gatchel et al., 2007). Assim sendo, a TCC parece ser uma escolha terapêutica coerente, por meio da qual crenças relacionadas à catastrofização podem ser confrontadas.

Quanto à autoeficácia, o estudo de Byrne et al. (2014) sugere um efeito grande no aumento da autoeficácia possivelmente relacionado à intervenção em MBCE. Segundo Bandura (1994), a autoeficácia percebida é definida como as crenças que uma pessoa tem a respeito de sua capacidade em desempenhar conforme o que é esperado em atividades que influenciam eventos significativos de sua vida. O autor destaca que uma forte percepção de eficácia aumenta o bem-estar pessoal de diferentes formas. Pessoas com alta confiança em suas capa- 
cidades encaram as dificuldades como desafios a serem superados, em vez de enxergá-las como ameaças a serem evitadas (Bandura, 1994).

Além disso, é comum que as reações fisiológicas ao estresse sejam interpretadas como sinais de propensão a um mau desempenho, sendo a reestruturação de interpretações distorcidas a respeito do próprio estado fisiológico uma das formas de promover a percepção de autoeficácia (Bandura, 1994). Dessa maneira, mais importante do que a intensidade das reações emocionais e físicas é a forma como elas são interpretadas (Bandura, 1994). Assim sendo, as práticas de atenção plena possuem um papel importante na observação das sensações corporais e das emoções sem julgamento, o que estaria diretamente relacionado à percepção de autoeficácia como encontrado por Byrne et al. (2014).

É possível que os protocolos de tratamento baseados na TCC para o medo do parto sejam mais eficazes se adaptados para o contexto social e cultural dos países em que serão aplicados. Nenhum dos estudos apresentados nesta pesquisa problematizou a universalidade dos protocolos utilizados. No Brasil, como já mencionado, devido às altas taxas de violência obstétrica e cirurgia cesariana, o medo de ser vítima de uma intervenção desnecessária (como uma episiotomia, ou uso de ocitocina sintética, de rotina) não caracterizaria uma distorção cognitiva, por exemplo. Dessa forma, intervenções aplicadas no Brasil poderiam avaliar os benefícios das técnicas de solução de problemas, que não foram abordadas nos estudos apresentados.

Para as mulheres atendidas na rede pública de saúde, não há garantia de que a assistência ao parto será oferecida por um profissional de saúde de sua confiança, já que o atendimento é realizado pela equipe médica de plantão. Assim, o Plano de Parto e Nascimento (Suárez-Cortés et al., 2015) pode ser uma opção importante para que a mulher comunique de forma assertiva o que ela espera do atendimento que irá receber. O Plano de Parto e Nascimento pode ser elaborado pela mulher após o recebimento das informações sobre a gravidez e o parto. Nesse documento, a mulher passa a registrar quais alternativas dentro do espectro de uma boa prática, sob condições normais, melhor contemplam seus desejos, suas expectativas, seus valores e suas necessidades. O documento tem o intuito de orientar a atenção de saúde prestada durante todo o processo de nascimento (Suárez-Cortés et al., 2015) e estimula maior confiança e sensação de controle relacionada ao parto. A percepção de estar no controle de si mesma, do próprio corpo e das ações dos profissionais de saúde sobre o seu corpo está positivamente relacionada à maior satisfação com o parto (Green, 1990).

Resumidamente, nesta revisão, as pesquisas que tiveram como desenho o estudo clínico randomizado (Larsson et al., 2017; Rondung et al., 2018) foram prejudicadas pelas perdas amostrais e não chegaram aos resultados esperados. Essa dificuldade de adesão ao tratamento coloca em risco a efetividade da intervenção do tipo iTCC para a população estudada. O estudo de Uçar e Golbasi (2018) apontou alguns benefícios do programa educacional baseado 
na TCC realizado em grupo, mas as considerações metodológicas apontadas tornam os resultados difíceis de ser interpretados. Já os estudos de Byrne et al. (2014), Nieminen et al. (2015) e Nieminen et al. (2016) não possibilitam uma atribuição de causalidade entre o tratamento proposto e a melhora do medo do parto, mas sugerem possíveis benefícios das intervenções em TCC para as mulheres grávidas.

É importante ressaltar que a falta de informação baseada em evidências sobre o parto, uma postura medicalizante que reforça o desequilíbrio da relação de poder entre a equipe obstétrica e a mulher, e a aquisição de informações trágicas sobre o trabalho de parto facilitam a percepção do parto como um evento perigoso (Melender, 2002; Pereira et al., 2011; Striebich et al., 2018). Nesse caso, a TCC poderia ajudar na construção de uma visão mais realista do parto (Nieminen et al., 2015), por ser uma abordagem que auxilia a formação do pensamento a partir de fatos e evidências (Beck, 2007). Contudo, enquanto a realidade da atenção obstétrica estiver pautada na falta de informação oferecida à mulher, na ausência de uma escuta empática das necessidades da parturiente e na prática de violência obstétrica, o medo relacionado ao parto estará estruturalmente fundamentado.

O medo do parto também está relacionado às cognições e experiências de vida de cada mulher. Por isso, técnicas terapêuticas como a reestruturação cognitiva, a exposição e a atenção plena demonstram ser relevantes para a elaboração de uma percepção menos catastrófica do parto e para o desenvolvimento de maior confiança nas próprias habilidades de enfrentamento do trabalho de parto. Sugerimos que estudos futuros investiguem a eficácia de cada uma dessas técnicas sobre a redução do medo do parto de forma individualizada. Dentro das limitações desta revisão sistemática, que incluem seu caráter descritivo e exploratório, conclui-se que a TCC é uma intervenção com potencial benefício para a redução do medo do parto. Contudo, novos estudos clínicos randomizados são necessários para que a TCC possa ser considerada eficaz no tratamento desse medo. 


\section{Referências}

American Psychological Association. Division 12. (2016). Exposure therapies for specific phobias status: Strong research support. https://www.div12.org/treatment/exposure-therapies-for-specificphobias/

Areskog, B., Uddenberg, N., \& Kjessler, B. (1981). Fear of childbirth in late pregnancy. Gynecologic and Obstetric Investigation, 12(5), 262-266. https://doi.org/10.1159/000299611

Bandura, A. (1994). Self-efficacy. In V. S. Ramachaudran (Ed.), Encyclopedia of human behavior (Vol. 4, pp. 71-81). Academic Press.

Beck, J. S. (2007). Terapia cognitiva: Teoria e prática. Artmed.

Berger, T. (2016). Intervenções autoguiadas baseadas na internet: Uma entrevista com o Dr. Thomas Berger [Entrevista concedida a Rodrigo T. Lopes]. Revista Brasileira de Terapias Cognitivas, 12(1), 57-61. https://doi.org/10.5935/1808-5687.20160009

Bewley, S., \& Cockburn, J. (2002). Responding to fear of childbirth. The Lancet, 359(9324), 21282129. https://doi.org/10.1016/S0140-6736(02)09113-4

Brasil. (2012). Cadernos da atenção básica: Atenção ao pré-natal de baixo risco. Ministério da Saúde, Secretaria de Atenção à Saúde, Departamento de Atenção Básica. http://bvsms.saude.gov.br/bvs/ publicacoes/cadernos_atencao_basica_32_prenatal.pdf

Byrne, J., Hauck, Y., Fisher, C., Bayes, S., \& Schutze, R. (2014). Effectiveness of a mindfulness-based childbirth education pilot study on maternal self-efficacy and fear of childbirth. Journal of Midwifery \& Women's Health, 59(2), 192-197. https://doi.org/10.1111/jmwh.12075

Gatchel, R. J., Peng, Y. B., Peters, M. L., Fuchs, P. N., \& Turk, D. C. (2007). The biopsychosocial approach to chronic pain: Scientific advances and future directions. Psychological Bulletin, 133(4), 581-624. https://doi.org/10.1037/0033-2909.133.4.581

Ghazaie, M., Davoodi, I., Neysi, A., Mehrabizadeh, H. M., \& Bassak Nejad, S. (2016). The effectiveness of cognitive-behavioral therapy on fear of childbirth, fear of pain, self-efficacy of childbirth and tendency to cesarean in nulliparous women. Iranian J. Obstet. Gynecol. Infertil., 19(31), 1-12. https://doi.org/10.22038/ijogi.2016.7989

Green, J. M. (1990). "Who is unhappy after childbirth?": Antenatal and intrapartum correlates from a prospective study. Journal of Reproductive and Infant Psychology, 8(3), 175-183. https://doi. org/10.1080/02646839008403622

Hofberg, K., \& Brockington, I. (2000). Tokophobia: An unreasoning dread of childbirth: A series of 26 cases. British Journal of Psychiatry, 176(1), 83-85. https://doi.org/10.1192/bjp.176.1.83

Hofberg, K., \& Ward, M. R. (2003). Fear of pregnancy and childbirth. Postgraduate Medical Journal, 79(935), 505-510. https://doi.org/10.1136/pmj.79.935.505

Hughes, A., Williams, M., Bardacke, N., Duncan, L. G., Dimidjian, S., \& Goodman, S. H. (2009). Mindfulness approaches to childbirth and parenting. British Journal of Midwifery, 17(10), 630-635. https://doi.org/10.12968/bjom.2009.17.10.44470

Kabat-Zinn, J., Lipworth, L., Burncy, R., \& Sellers, W. (1986). Four-year follow-up of a meditation-based program for the self-regulation of chronic pain: Treatment outcomes and compliance. 
The Clinical Journal of Pain, 2(3), 159-774. https://doi.org/10.1097/00002508-19860203000004

Larsson, B., Karlström, A., Rubertsson, C., Ternström, E., Ekdahl, J., Segebladh, B., \& Hildingsson, I. (2017). Birth preference in women undergoing treatment for childbirth fear: A randomised controlled trial. Women and Birth, 30(6), 460-467. https://doi.org/10.1016/j.wombi. 2017.04.004

Leal, M. C., Torres, J. A., Domingues, R. M. S. M., Theme Filha, M. M., Bittencourt, S., Dias, M. A. B., Pereira, M. N., Viellas, E. F., Diniz, C. S. G., d’Orsi, E., Silva, A. A. M., Pereira, A. P. E. Bastos, M. H., Moreira, M. E. L., Lansky, S., \& Gama, S. G. N. da (2012). Nascer no Brasil: Sumário executivo temático da pesquisa. CCI/ENSP. http://www.ensp.fiocruz.br/portal-ensp/informe/site/arquivos/ anexos/nascerweb.pdf

Melender, H.-L. (2002). Experiences of fears associated with pregnancy and childbirth: A study of 329 pregnant women. Birth: Issues in Perinatal Care, 29(2), 101-111. https://doi.org/ 10.1046/j.1523-536X.2002.00170.x

Moher, D., Liberati, A., Tetzlaff, J., \& Altman, D. G., The PRISMA Group. (2009). Preferred reporting items for systematic reviews and meta-analyses: The prisma statement. PLoS Med., 6(7), 1-6. https://doi.org/10.1371/journal.pmed.1000097

Nieminen, K., Andersson, G., Wijma, B., Ryding, E.-L., \& Wijma, K. (2016). Treatment of nulliparous women with severe fear of childbirth via the Internet: A feasibility study. Journal of Psychosomatic Obstetrics \& Gynecology, 37(2), 37-43. https://doi.org/10.3109/0167482X.2016.1140143

Nieminen, K., Malmquist, A., Wijma, B., Ryding, E.-L., Andersson, G., \& Wijma, K. (2015). Nulliparous pregnant women's narratives of imminent childbirth before and after internet-based cognitive behavioural therapy for severe fear of childbirth: A qualitative study. BJOG: An International Journal of Obstetrics \& Gynaecology, 122(9), 1259-1265. https://doi.org/10.1111/1471-0528.13358

Nunes, R. D., Traebert, E., Seemann, M., \& Traebert, J. (2021). Tokophobia Assessment Questionnaire: A new instrument. Brazilian Journal of Psychiatry, 43(1), 112-114. https://doi.org/10.1590/ 1516-4446-2020-1252

O'Connell, M. A., Leahy-Warren, P., Khashan, A. S., Kenny, L. C., \& O'Neill, S. M. (2017). Worldwide prevalence of tocophobia in pregnant women: Systematic review and meta-analysis. Acta Obstet. Gynecol. Scand., 96, 907-920. https://doi.org/10.1111/aogs.13138

Olatunji, B.O., Cisler J. M., \& Deacon B. J. (2010). Efficacy of cognitive behavioral therapy for anxiety disorders: A review of meta-analytic findings. Psychiatr Clin North Am, 33(3), 557-577. https:// doi.org/10.1016/j.psc.2010.04.002

Pereira, R. R., Franco, S. C., \& Baldin, N. (2011). Pain and the protagonism of women in parturition. Revista Brasileira de Anestesiologia, 61(3), 382-388. https://doi.org/10.1590/So03470942011000300014

Ryding, E. L. (2017). Medo do parto: Definição e diagnóstico. In U. Björklund \& E. L. Ryding (Ed.), Medo do parto (pp. 7-14). Sociedade Sueca de Obstetrícia e Ginecologia. https://www.sfog.se/natupplaga/1005623_A2fb46497-d45b-466d-8126-c58e99fe856d.pdf

Rondung, E., Ternström, E., Hildingsson, I., Haines, H. M., Sundin, Ö., Ekdahl, J., Karlström, A., Larsson, B.; Segeblad, B., Baylis, \& R., Rubertsson, C. (2018). Comparing internet-based cognitive 
behavioral therapy with standard care for women with fear of birth: Randomized controlled trial. JMIR Ment. Health, 5(3), 1-16. https://doi.org/10.2196/10420

Rondung, E., Thomtén, J., \& Sundin, Ö. (2016). Psychological perspectives on fear of childbirth. Journal of Anxiety Disorders, 44, 80-91. https://doi.org/10.1016/j.janxdis.2016.10.007

Sampaio, R. F., \& Mancini, M. C. (2007). Estudos de revisão sistemática: Um guia para síntese criteriosa da evidência científica. Brazilian Journal of Physical Therapy, 11(1), 83-89. https://doi. org/10.1590/S1413-35552007000100013

Souza, A. S. R., Amorim, M. M. R., \& Porto, A. M. F. (2010). Condições frequentemente associadas com cesariana, sem respaldo científico. FEMINA, 38(10), 505-516. http://files.bvs.br/upload/S/ 0100-7254/2010/v38n10/a1708.pdf

Striebich, S., Mattern, E., \& Ayerle, G. M. (2018). Support for pregnant women identified with fear of childbirth (FOC)/tokophobia: A systematic review of approaches and interventions. Midwifery, 61, 97-115. https://doi.org/10.1016/j.midw.2018.02.013

Suárez-Cortés, M., Armero-Barranco, D., Canteras-Jordana, M., \& Martínez-Roche, M. E. (2015). Use and influence of delivery and birth plans in the humanizing delivery process. Rev. Latino-Am. Enferm., 23(3), 520-526. https://doi.org/10.1590/0104-1169.0067.2583

Ternström, E. (2018). Identification and treatment of women with a fear of birth. [Ubpublsihed Doctoral Thesis]. Universidade de Uppsala. http://uu.diva-portal.org/smash/record. jsf?pid=diva2:1196389

Uçar, T., \& Golbasi, Z. (2018). Effect of an educational program based on cognitive behavioral techniques on fear of childbirth and the birth process. Journal of Psychosomatic Obstetrics \& Gynecology, 40(2) 1-19. https://doi.org/10.1080/0167482X.2018.1453800

Vandenberghe, L., \& Sousa, A. C. A. (2006). Mindfulness nas terapias cognitivas e comportamentais. Revista Brasileira de Terapias Cognitivas, 2(1), 35-44. http://pepsic.bvsalud.org/scielo.php? script=sci_arttext $\&$ pid $=$ S1808 -56872006000100004

Venturi, G., Bokany, V., \& Dias, R. (2010). Mulheres brasileiras e gênero nos espaços público e privado. Fundação Perseu Abramo \& Serviço Social do Comércio. https://fpabramo.org.br/publicacoes/ publicacao/pesquisa-mulheres-brasileiras-e-genero-nos-espacos-publico-e-privado-2010/

World Health Organization. (2015). Declaração da OMS sobre Taxas de Cesáreas. WHO. https://apps.who. int/iris/bitstream/handle/10665/161442/WHO_RHR_15.02_por.pdf?sequence=3

World Health Organization. (2019). Trends in maternal mortality 2000 to 2017: Estimates by WHO, UNICEF, UNFPA, World Bank Group and the United Nations Population Division: Executive summary. WHO. https://apps.who.int/iris/handle/10665/327596

Wijma, K., Wijma, B., \& Zar, M. (1998). Psychometric aspects of the W-DEQ: A new questionnaire for the measurement of fear of childbirth. Journal of Psychosomatic Obstetrics \& Gynecology, 19(2), 84-97. https://doi.org/10.3109/01674829809048501

Zorzam, B., \& Cavalcanti, P. (2017). Direitos das mulheres no parto: Conversando com profissionais da saúde e do direito (Cartilha). Coletivo Feminista de Sexualidade e Saúde. https://www.mulheres. org.br/wp-content/uploads/2020/02/direito-mulheres-parto.pdf 
EQUIPE EDITORIAL

Editora-chefe

Cristiane Silvestre de Paula

\section{Editores associados}

Alessandra Gotuzo Seabra

Ana Alexandra Caldas Osório

Luiz Renato Rodrigues Carreiro

Maria Cristina Triguero

Veloz Teixeira

\section{Editores de seção}

"Avaliação Psicológica"

Alexandre Serpa

André Luiz de Carvalho

Braule Pinto

Luiz Renato Rodrigues Carreiro

Vera Lúcia Esteves Mateus

"Psicologia e Educação"

Alessandra Gotuzo Seabra

Carlo Schmidt

Regina Basso Zanon
"Psicologia Social e Saúde das

Populações"

Enzo Banti Bissoli

Marina Xavier Carpena

"Psicologia Clínica"

Carolina Andrea Ziebold Jorquera

Julia Garcia Durand

Natalia Becker

\section{"Desenvolvimento Humano"}

Maria Cristina Triguero

Veloz Teixeira

Rosane Lowenthal

\section{Suporte técnico}

Maria Fernanda Liuti

Bento da Silva

Camila Fragoso Ribeiro
PRODUÇÃO EDITORIAL

Coordenação editorial

Ana Claudia de Mauro

Estagiários editoriais

Júlia Lins Reis

Élcio Carvalho

Pietro Menezes

Preparação de originais

Carlos Villarruel

\section{Revisão}

Vera Ayres

\section{Diagramação}

Acqua Estúdio Gráfico 